\title{
SPIN REPRESENTATIONS WITH NEGATIVE INDICES
}

\author{
MADLINE AL-TAHAN, MOHAMMAD N. ABDULRAHIM AND SAMER S. HABRE
}

\begin{abstract}
We consider the spin representation of Artin's braid group, which has a negative index of one and was originally given by D. D. Long and explicitly computed by J.P.Tian. In our work, we find sufficient conditions under which the complex specialization of that representation, namely $\alpha: B_{n} \rightarrow G L_{n^{2}}(\mathbb{C})$, is unitary relative to a nonsingular hermitian matrix.
\end{abstract}

\section{Introduction}

The braid group, $B_{n}$, has a well- known representation due to $\operatorname{Artin}$ in the group $\operatorname{Aut}\left(F_{n}\right)$ of automorphisms of the free group $F_{n}$ generated by $x_{1}, \ldots, x_{n}$. The automorphism corresponding to the braid generator $\sigma_{i}$ takes $x_{i}$ to $x_{i} x_{i+1} x_{i}^{-1} ; x_{i+1}$ to $x_{i}$, and fixes all other free generators. Applying the Fox derivatives and Magnus representation, we get the classical Burau representation which is of degree $n$. Given a compact semisimple Lie group $G$ and a free group $F_{n}$ of rank $n$, D. D. Long constructed a new representation of the braid group by letting the automorphisms of the free group $F_{n}$ act on the representation variety $R=R\left(F_{n}, G\right)$ [3]. J.P.Tian has made explicit computations when $G=S U(2, \mathbb{C})$, the group of unitary matrices of determinant 1 [6]. It was shown that the Weyl group $W\left(G^{n}\right)$ is $\mathbb{Z}_{2}{ }^{n}$ and it is has a one-to-one correspondence with the set of all spins $\left(s_{1}, s_{2}, \ldots, s_{n}\right)$. These spins are the immediate result of the action of the Weyl group $W(G)$ on $S^{1}$. For more details, see [6]. Generalizations to include subgroups of $B_{n}$ were made by S.Bigelow and J. P. Tian [1]. Each of the components of the maximal torus $S^{1} \times S^{1} \times \ldots S^{1}$ of $S U(2, \mathbb{C})^{n}$ is given an orientation with a number $s_{i}= \pm 1$, $i=1,2, \ldots, n$. The set $\left(s_{1}, s_{2}, \ldots, s_{n}\right)$ is called the spin $S$. The cardinality of the set $\left\{s_{i} \mid s_{i}=-1\right\}$ gives the negative index $b(S)$. When $b(S)=0$ or $b(S)=n$, the spin representations are simply the classical Burau representation after we replace $\lambda^{2}$ by $t$ and $t^{-1}$ respectively. In our work, we consider the case $b(S)=1$, where its corresponding representation is of degree $n^{2}$.

In Section 2, we define the complex specialization of the spin representation with the negative index $b(S)=1$, namely $\alpha(z): B_{n} \rightarrow G L_{n^{2}}(\mathbb{C})$. Here, $z$ is a complex number on the

Received July 16, 2013, accepted April 23, 2014.

2010 Mathematics Subject Classification. 20 F36.

Key words and phrases. Braid group, Burau representation, unitary.

Corresponding author: Mohammad N. Abdulrahim. 
unit circle. It is well known that the problem of identifying the images of the different linear representations of the braid group and its subgroups was always important in the theory of braid group representations. The answer to such a problem would provide a tool to attack the question of whether or not these representations are faithful. Examples of unitary representations include the Burau representation of the braid group and the Gassner repesentation of the pure braid group, a nomal subgroup of the braid group. For more details, see [5]. In section 3, we prove our main theorem, Theorem 5 , which shows that the spin representation of the braid group, $B_{n}$, with the spins of $b(S)=1$, is unitary relative to a nonsingular hermitian matrix if $z= \pm 1$ or $z^{n-1} \neq 1$.

\section{Definitions}

Definition 1 ([2]). The braid group on $n$ strings, $B_{n}$, is the abstract group with presentation

$$
\begin{gathered}
B_{n}=\left\langle\sigma_{1}, \ldots, \sigma_{n-1}\right| \sigma_{i} \sigma_{i+1} \sigma_{i}=\sigma_{i+1} \sigma_{i} \sigma_{i+1} \text { for } i=1, \ldots, n-2, \\
\left.\sigma_{i} \sigma_{j}=\sigma_{j} \sigma_{i} \text { if }|i-j| \geq 2\right\rangle .
\end{gathered}
$$

The generators $\sigma_{1}, \ldots, \sigma_{n-1}$ are called the standard generators of $B_{n}$.

Applying the new derivative of the Burau representation adopted by D. D. Long in [3], J. P. Tian has made explicit computations for such a representation [6]. Hence, we introduce the following definition.

Definition $2([3,6])$. Given a non zero complex number on the unit circle, namely $\lambda$. The complex specialization of the spin representation of the braid group $\alpha(\lambda): B_{n} \rightarrow G L_{n^{2}}(\mathbb{C})$, with the negative index of one, is defined as follows:

For $1 \leq k \leq n-1$,

$$
\begin{gathered}
\tau_{k}=\underbrace{G_{k} \oplus \cdots \oplus G_{k}}_{k-1} \oplus\left(\begin{array}{cc}
0 & F_{k} \\
E_{k} & 0
\end{array}\right) \oplus \underbrace{G_{k} \oplus \cdots \oplus G_{k}}_{n-k-1}, \text { where } \\
G_{k}=I_{k-1} \oplus\left(\begin{array}{cc}
1-\lambda^{2} & \lambda^{2} \\
1 & 0
\end{array}\right) \oplus I_{n-k-1}, \\
F_{k}=I_{k-1} \oplus\left(\begin{array}{cc}
1-\lambda^{-2} & \lambda^{2} \\
1 & 0
\end{array}\right) \oplus I_{n-k-1},
\end{gathered}
$$

and

$$
E_{k}=I_{k-1} \oplus\left(\begin{array}{cc}
1-\lambda^{2} & \lambda^{-2} \\
1 & 0
\end{array}\right) \oplus I_{n-k-1} .
$$


Here, we mean by $\tau_{k}$ the image of the braid generator under the spin representation with the spins of $b(S)=1$. For simplicity, we denote $\lambda^{2}$ by $z$.

It is clear that the matrices corresponding to $\tau_{k}$ 's satisfy the relations among the braid group generators.

\section{Spin Representations with $b(S)=1$ Are Unitary}

Notation 3. Let $(*): M_{m}\left(\mathbb{C}\left[t^{ \pm 1}\right]\right)$ be an involution defined as follows:

$$
\left(f_{i j}(t)\right)^{*}=f_{j i}\left(t^{-1}\right), f_{i j}(t) \in \mathbb{C}\left[t^{ \pm 1}\right] .
$$

Definition 4. Let $H$ and $U$ be elements of $G L_{n^{2}}(\mathbb{C}) . U$ is called unitary (relative to $H$ ) if $U H U^{*}=H$.

Having $z=\lambda^{2}$, a non zero complex number on the unit circle, we determine sufficient conditions for $z$ under which the spin representation of the braid group, having the negative index of one, is unitary.

Theorem 5. Let $z$ be a complex number on the unit circle. The spin representation of the braid $\operatorname{group} \alpha(z): B_{n} \rightarrow G L_{n^{2}}(\mathbb{C})$, with the negative index $b(S)=1$, is unitary relative to a nonsingular hermitian matrix if $z= \pm 1$ or $z^{n-1} \neq 1$.

Proof. We distinguish between three cases.

Case 1. $z=1$. It is clear that $\tau_{k} \tau_{k}^{*}=I_{n^{2}}$ for every $k=1, \ldots, n-1$.

Case 2. $z=-1$. We easily verify that $\tau_{k} N \tau_{k}^{*}=N$, where $N=\oplus_{1}^{n} M$ and $M$ is the $n \times n$ nonsingular matrix given by $\left(m_{s, t}\right)$, where

$$
m_{s, t}= \begin{cases}1, & \text { if } s=t \\ 1-i, & \text { if } s<t \\ 1+i, & \text { if } s>t\end{cases}
$$

Here, $i$ is the complex number given by $i^{2}=-1$.

Case 3. $z^{n-1} \neq 1$. If $z=-1$ then we return to Case 2. Otherwise, we assume that $z \neq-1$. Let $N$ be the matrix defined as $N=\oplus_{i=1}^{n} M_{i}$, where $M_{i}$ is the $n \times n$ matrix given by 


$$
\begin{gathered}
\left(\begin{array}{ccccccccc}
\frac{-(1+z)^{2}}{z} & -\frac{1+z}{z} & \ldots & -\frac{1+z}{z} & 1+z & -\frac{1+z}{z} & \ldots & \ldots & -\frac{1+z}{z} \\
-(1+z) & \ddots & & \vdots & \vdots & \vdots & & & \vdots \\
\vdots & & & & & & & & \\
-(1+z) & \ldots & \frac{-(1+z)^{2}}{z} & -\frac{1+z}{z} & 1+z & -\frac{1+z}{z} & \ldots & \ldots & -\frac{1+z}{z} \\
-(1+z) & \ldots & -(1+z) & \frac{-(1+z)^{2}}{z} & 1+z & -\frac{1+z}{z} & \ldots & \ldots & -\frac{1+z}{z} \\
\frac{1+z}{z} & \ldots & \ldots & \frac{1+z}{z} & \underbrace{0}_{(i, i)^{t h}} & \frac{1+z}{z^{2}} & \ldots & \ldots & \frac{1+z}{z^{2}} \\
-(1+z) & \ldots & \ldots & -(1+z) & z(1+z) & \frac{-(1+z)^{2}}{z} & -\frac{1+z}{z} & \ldots & -\frac{1+z}{z} \\
-(1+z) & & & & & -(1+z) & \ddots & & \vdots \\
\vdots & & & \vdots & & & \ddots & -\frac{1+z}{z} \\
-(1+z) & \ldots & \ldots & -(1+z) & z(1+z) & -(1+z) & \ldots & -(1+z) & \frac{-(1+z)^{2}}{z}
\end{array}\right), \\
i=1, \ldots, n .
\end{gathered}
$$

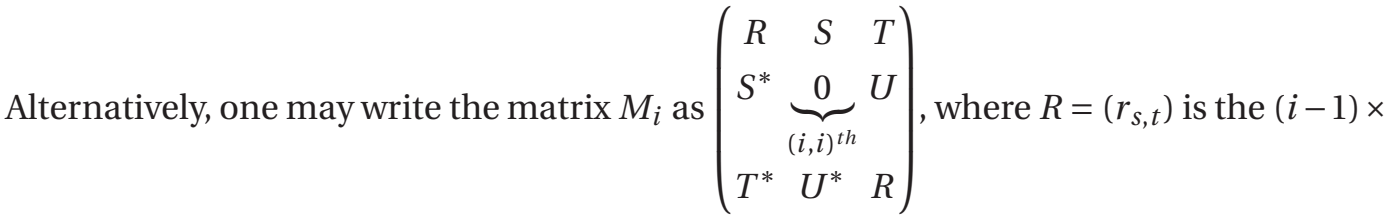
$(i-1)$ matrix given by

$$
r_{s t}=\left\{\begin{array}{cc}
\frac{-(1+z)^{2}}{z}, & \text { if } s=t \\
\frac{-(1+z)}{z}, & \text { if } s<t \\
-(1+z), & \text { if } s>t
\end{array}\right.
$$

Also, the matrix $S$ is the $(i-1)$ column matrix with $1+z$ as its entries, $T$ is the $(i-1) \times(n-i)$ matrix with $\frac{-(1+z)}{z}$ as its entries and $U$ is the $n-i$ row matrix with $\frac{1+z}{z^{2}}$ as its entries.

Having that $\operatorname{det}\left(M_{i}\right)=\left(-z^{-1}\right)^{n-1} \frac{(1+z)^{n}\left(1-z^{n-1}\right)}{(1-z)} \neq 0$ by our hypothesis, we get that $M_{i}$ is an invertible hermitian matrix for every $i=1, \ldots, n$. This implies that that $N$ is also hermitian and invertible. Under direct computations, we have that for $1 \leq k \leq n-1$

$$
\tau_{k} N \tau_{k}^{*}=G_{k} M_{1} G_{k}^{*} \oplus \ldots \oplus G_{k} M_{k-1} G_{k}^{*} \oplus F_{k} M_{k+1} F_{k}^{*} \oplus E_{k} M_{k} E_{k}^{*} \oplus G_{k} M_{k+2} G_{k}^{*} \oplus \ldots \oplus G_{k} M_{n} G_{k}^{*} .
$$

We show that $G_{k} M_{i} G_{k}^{*}=M_{i}$ for $i \in[1, k-1] \cup[k+2, n]$. We also show that $F_{k} M_{k+1} F_{k}^{*}=$ $M_{k}$ and $E_{k} M_{k} E_{k}^{*}=M_{k+1}$.

For simplicity, we set $g=\frac{1+z}{z}$. Throughout the following work, we will only write the entries of the $k^{t h}$ and the $(k+1)^{t h}$ rows in the matrices $M_{i}$, and all other entries from different rows will be denoted by $*$ since they remain unchanged after performing the matrix multiplication. 
We now prove that $G_{k} M_{i} G_{k}^{*}=M_{i}$ for $1 \leq i \leq k-1$. The matrices $G_{k}$ and $M_{i}$ are given by

$$
G_{k}=\left(\begin{array}{ccccc}
I_{k-1} & & & \\
& 1-z & z & \\
& 1 & 0 & \\
& & & I_{n-k-1}
\end{array}\right)
$$

and

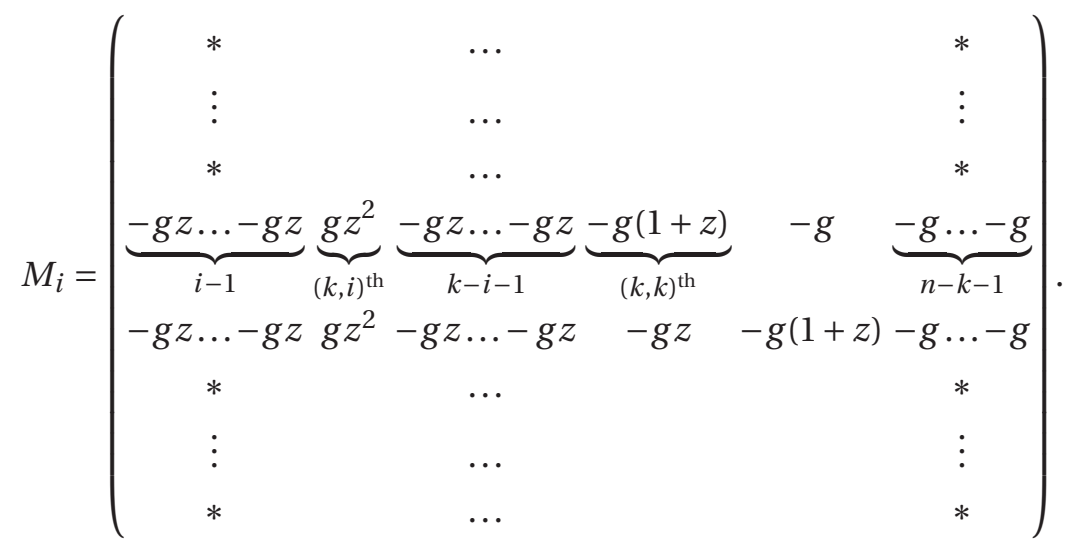

Direct computations show that

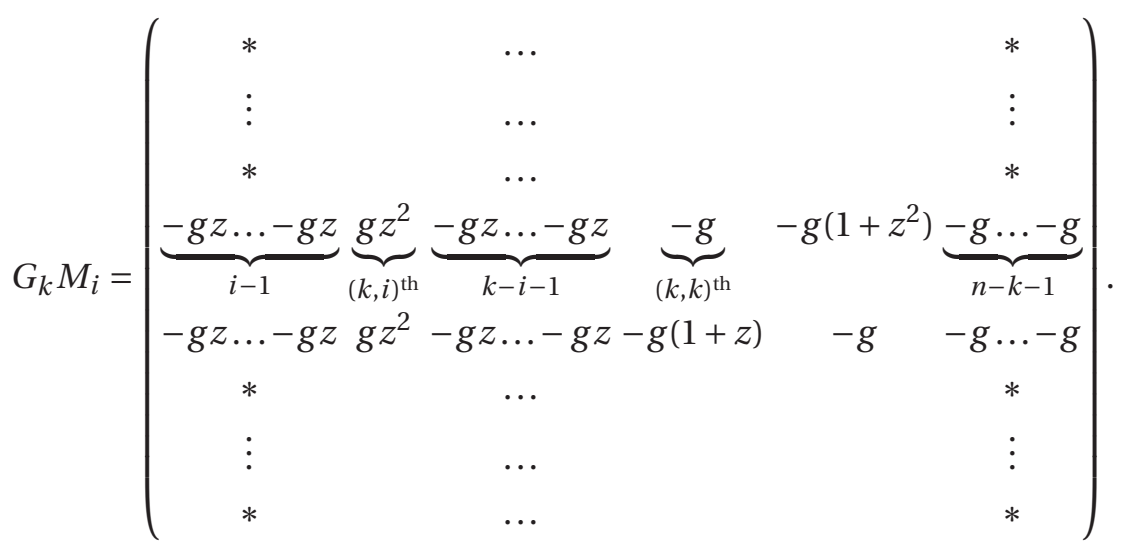

Using simple computations, it is easy to see that $G_{k} M_{i} G_{k}^{*}=M_{i}$ for $1 \leq i \leq k-1$. Likewise, we show that the equality also holds true for $k+2 \leq i \leq n$.

On the other hand, the matrices $F_{k}$ and $M_{k+1}$ are given by

$$
F_{k}=\left(\begin{array}{cccc}
I_{k-1} & & & \\
& 1-z^{-1} & z & \\
& 1 & 0 & \\
& & & I_{n-k-1}
\end{array}\right)
$$


and

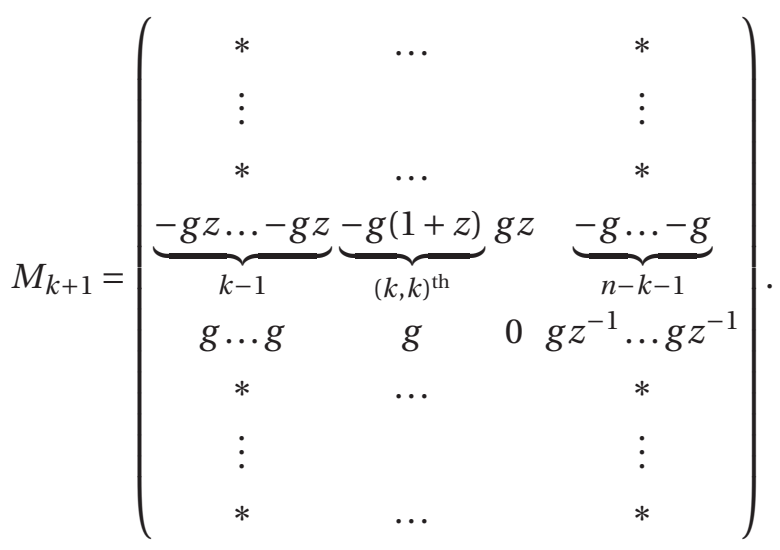

Direct computations show that

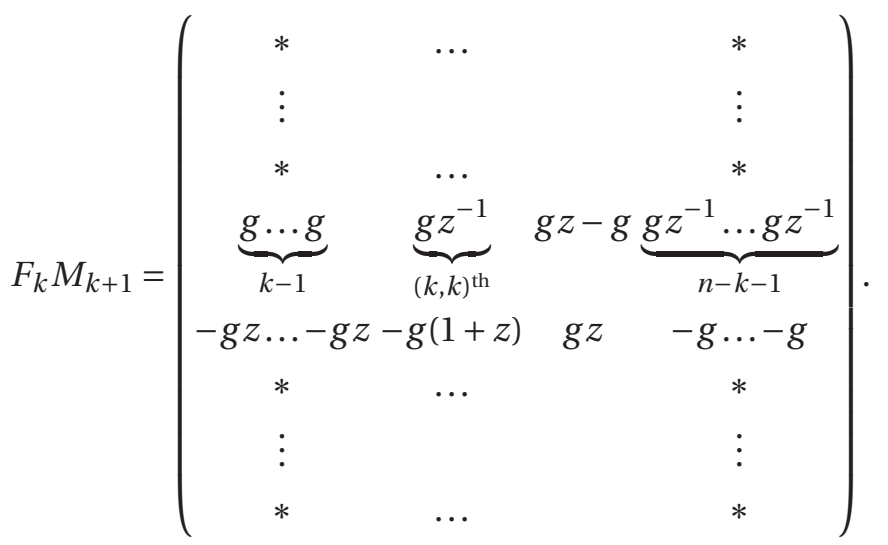

Using simple computations, it is now easy to verify that $F_{k} M_{k+1} F_{k}^{*}=M_{k}$.

Finally, the matrices $E_{k}$ and $M_{k}$ are given by

$$
E_{k}=\left(\begin{array}{cccc}
I_{k-1} & & & \\
& 1-z & z^{-1} & \\
& 1 & 0 & \\
& & & I_{n-k-1}
\end{array}\right)
$$


and

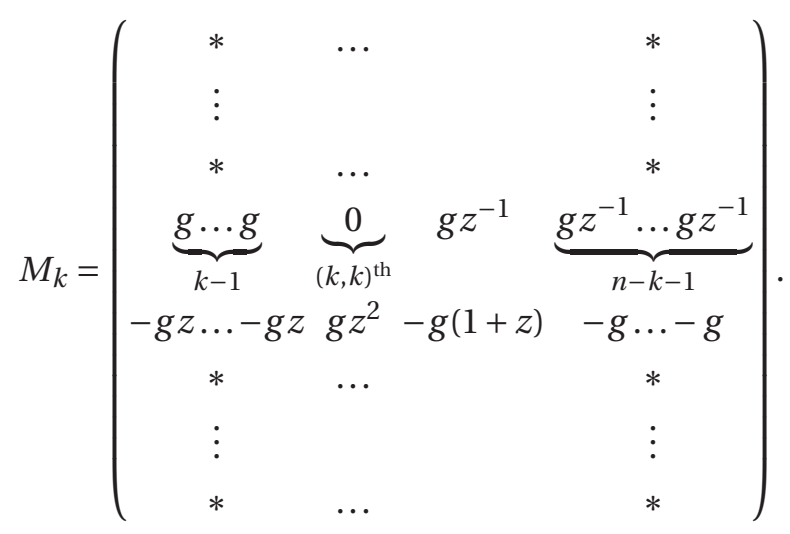

Thus, we get that

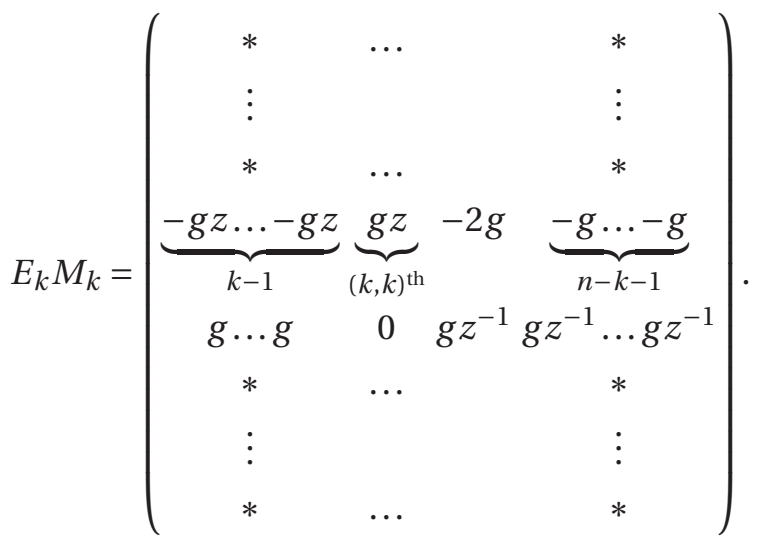

Again, it is easy to verify that $E_{k} M_{k} E_{k}^{*}=M_{k+1}$.

We then conclude that $\tau_{k} N \tau_{k}{ }^{*}=N$ and so the proof is completed.

Remark 6. It is easy to see, by Theorem 5, that for $n=2$ and $n=3, \alpha(z): B_{n} \rightarrow G L_{n^{2}}(\mathbb{C})$ is unitary relative to a nonsingular hermitian matrix for all complex numbers $z$ on the unit circle.

\section{References}

[1] S. Bigelow and J. P. Tian, Generalized Long-Moody representations of braid groups, Communications in Contemporary Math., 10 (2008) (suppl.1), 1093-1102.

[2] J. S. Birman, Braids, Links and Mapping Class Groups, Annals of Mathematical Studies, Princeton University Press, volume 82, New Jersey, 1975.

[3] D. D. Long, On the linear representation of braid groups, Transaction of the American Mathematical Society, 311 (1989), 535-560. 
[4] V. Shpilrain, Representing Braids by Automorphisms, International Journal of Algebra and Computation, 11 (2001), 773-777.

[5] C. C. Squier, The Burau representation is unitary. Proceedings of the American Mathematical Society, 90(1984), 199-202.

[6] J. P. Tian, Private communications, 2007.

Department of Mathematics, Beirut Arab University, P.O. Box 11-5020, Beirut, Lebanon.

E-mail: maa531@bau.edu.lb

Department of Mathematics, Beirut Arab University, P.O. Box 11-5020, Beirut, Lebanon.

E-mail:mna@bau.edu.lb

Department of Computer Science and Mathematics, Lebanese American University, P.O. Box 13-5053 Chouran, Beirut, Lebanon.

E-mail: shabre@lau.edu.lb 